\title{
MAXIMAL PRIME DIVISORS IN ARITHMETICAL RINGS
}

\author{
LASZLO FUCHS, WILLIAM HEINZER, AND BRUCE OLBERDING
}

\begin{abstract}
We investigate for an ideal $A$ of an arithmetical ring $R$ the relationship between the set $\operatorname{Max}(A)$ of maximal prime divisors of $A$ and the set $\mathcal{X}_{A}$ of maximal members of the set of Krull associated primes of $A$. We show that the arithmetical rings $R$ such that $\mathcal{X}_{A} \subseteq \operatorname{Max}(A)$ for every regular ideal $A$ are precisely those satisfying the "strong" separation property. For a Prüfer domain $R$, we prove that every branched prime ideal of height greater than one is the radical of a finitely generated ideal if and only if $\operatorname{End}(A)_{M}=\operatorname{End}\left(A_{M}\right)$ for every nonzero ideal $A$ and maximal ideal $M$ of $R$. We use this to prove that if in addition $R$ is a QR-domain, then every maximal prime divisor of an ideal $A$ of $R$ is a Krull associated prime of $A$ (i.e. $\mathcal{X}_{A}=\operatorname{Max}(A)$ ) if and only if each branched prime ideal of $R$ of height greater than one is the radical of a finitely generated ideal.
\end{abstract}

\section{INTRODUCTION}

Let $R$ be a commutative ring with identity. Associated to a proper ideal $A$ of $R$ is the set $S(A)=\{x \in R: x y \in A$ for some $y \in R \backslash A\}$ of elements of $R$ that are non-prime to $A$. The complement $R \backslash S(A)$ of elements prime to $A$ is closed under multiplication. Krull proves [14, page 732$]$ that ideals maximal with respect to not meeting a multiplicatively closed set are prime ideals and defines the maximal prime divisors of $A$ as the prime ideals $P$ of $R$ that contain $A$ and are maximal with respect to the property $P \subseteq S(A)$.

Let $\operatorname{Max}(A)$ denote the set of maximal prime divisors of $A$. For $P \in \operatorname{Max}(A)$, Krull defines the ideal $A_{(P)}=\{x \in R: x y \in A$ for some $y \in R \backslash P\}$ to be the principal component of $A$ with respect to $P$ and establishes the decomposition of every proper ideal $A$ of $R$ as the intersection of its principal components $A=$ $\bigcap_{P \in \operatorname{Max}(A)} A_{(P)}[14$, Satz 2]. However, as we discuss in [4], a drawback to this decomposition is that we do not know what kind of ideals the $A_{(P)}$ are. For instance, there sometimes exist elements of $P$ that are prime to the principal component $A_{(P)}$; indeed, the ideal $A_{(P)}$ is not in general a primal ideal, where an ideal $B$ is said to be primal if the set $S(B)$ of elements non-prime to $B$ is an ideal. If $B$ is primal, then $S(B)$ is a prime ideal called the adjoint prime of $B$.

1991 Mathematics Subject Classification. Primary 13A15; Secondary 13 F05.

Key words and phrases. Primal ideal, associated prime, arithmetical ring, Prüfer domain. 
To obtain in a commutative ring without finiteness conditions a decomposition of the ideal $A$ that involves components closely tied to $A$ with structure we know, in [4] we define the set $\mathcal{X}_{A}$ of maximal Krull associated primes of $A$ and obtain using the set $\mathcal{X}_{A}$ a canonical primal decomposition of $A$.

If $R$ is a Noetherian ring and $\operatorname{Ass}(A)=\left\{P_{1}, P_{2}, \ldots, P_{n}\right\}$ is the set of associated primes of a proper ideal $A$ of $R$, then $S(A)=\bigcup_{i=1}^{n} P_{i}$. It follows that the maximal prime divisors of $A$ are exactly the prime ideals of $R$ that are maximal members of $\operatorname{Ass}(A)$. In this sense, the maximal prime divisors of an ideal of a Noetherian ring are well-understood.

However if $R$ is not Noetherian, the set $\operatorname{Max}(A)$ of maximal prime divisors of $A$ is generally less transparent, and the primes in $\operatorname{Max}(A)$ need not be "associated." There are several inequivalent notions of an associated prime of an ideal of a general commutative ring, but from our point of view, it is the Krull associated primes that are most natural. We review this and related notions in Section 1. Motivated by the Noetherian case, we are thus interested in the question of when the set of maximal prime divisors of an ideal $A$ coincides with the set of maximal members of the set of Krull associated primes of $A$. We shall examine this question for the class of arithmetical rings, those rings $R$ such that for every maximal ideal $M$ of $R, R_{M}$ is a valuation ring, that is, a ring for which the set of ideals is linearly ordered by inclusion. Of particular interest is the class of Prüfer domains, namely the arithmetical integral domains.

Our purpose in the present paper is to investigate for ideals $A$ of an arithmetical ring or Prüfer domain the interrelationship between the sets $\operatorname{Max}(A)$ and $\mathcal{X}_{A}$. The connection between these two sets of prime ideals is of special interest in the context of arithmetical rings. Indeed, if $A$ is a proper ideal of an arithmetical ring $R$, then $\operatorname{Max}(A)=\mathcal{X}_{A}$ if and only if every prime ideal containing $A$ but not prime to $A$ is a Krull associated prime (Corollary 1.3). Also, Krull mentions [15, p. 16] that he does not know whether the principal components $A_{(P)}$ of $A, P \in \operatorname{Max}(A)$, are always $P$-primal ideals. (A primal ideal $B$ is $Q$-primal if $Q=S(B)$.) Examples show that the answer is in the negative (see [17],[5] and [4, Example 3.8]); we investigate in Section 3 arithmetical rings for which the answer to this question is in the affirmative for all regular ideals. (An ideal $A$ of $R$ is regular if it contains a regular element, i.e., an element that is not a zero-divisor.) This is also of special interest, because an ideal of an arithmetical ring is primal exactly if it is irreducible (Theorem 1.8 of [4]). 
In Section 3 we examine conditions on an arithmetical ring $R$ in order that each maximal prime divisor of a regular ideal $A$ of $R$ be a Krull associated prime of $A$. It follows from Theorem 2.3 that a necessary condition for this to hold is that $R$ satisfy the strong separation property: for regular prime ideals $P \subset Q$ and regular element $r \in P$, there exists $s \in Q$ such that $P \subset(r, s) R \subseteq Q$. We conclude that Prüfer domains such as the ring of entire functions and the $\operatorname{ring} \operatorname{Int}(\mathbb{Z})$ of integer-valued polynomials contain ideals $A$ having the property that there exists a maximal prime divisor of $A$ that is not a Krull associated prime of $A$.

For a regular ideal $A$ of an arithmetical ring, we consider in Theorem 3.7 conditions in order that the set of maximal prime divisors of $A$ is precisely the set of prime ideals that are maximal among the Krull associated primes of $A$. We deduce a complete characterization of the QR-domains with this property. (By contrast, ideals of Noetherian rings always exhibit equality between these two sets of prime ideals.)

The results that lay the groundwork for this theorem touch on several interesting technical aspects of arithmetical rings and Prüfer domains. In particular, there is a connection between our problem and that of when $\operatorname{End}(X)$ localizes, that is, when $\operatorname{End}(X)_{M}=\operatorname{End}\left(X_{M}\right)$ for a submodule $X$ of the quotient field of $R$ and maximal ideals $M$ of $R$.

Acknowledgement. We thank Gabriel Picavet and David Rush for helpful conversations on the topics of this paper and [4], and for showing us the connections of our work to the literature on Krull associated primes.

\section{Krull associated PRIMES}

In this section we briefly review the notion of a Krull associated prime of a proper ideal of a ring. We became interested in the Krull associated primes of an ideal because of their connection to the primal isolated components of the ideal. For a more complete treatment of these primes and their relation to issues involving the primal decompositions of an ideal, see [4].

Let $A$ be a proper ideal of the ring $R$. Following [12], we define a prime ideal $P$ of $R$ to be a Krull associated prime of the ideal $A$ if for every element $x \in P$, there exists $y \in R$ such that $x \in(A: y) \subseteq P$. A prime ideal $P$ of $R$ is called a weak-Bourbaki associated prime of $A$ if it is a minimal prime divisor of $(A: x)$ for some $x \in R \backslash A$. Following [11, page 279], we call $P$ a Zariski-Samuel associated prime of $A$ if $P=\sqrt{(A: x)}$ for some $x \in R \backslash A$. If $R$ is a Noetherian ring, then 
all three of these notions of an associated prime coincide, but for non-Noetherian rings these notions are in general distinct (see [11], for example).

It is clear that a Zariski-Samuel associated prime is a weak-Bourbaki associated prime. Moreover, it is noted in Lemma 2.1 of [4] that $P$ is a Krull associated prime of $A$ if and only if $P$ is a set-theoretic union of weak-Bourbaki primes of $A$. It is this characterization of Krull associated primes that we shall use in what follows.

We denote by $\operatorname{Ass}(A)$ the set of Krull associated primes of $A$. Notice that if $A$ is a proper ideal of $R$, then $\operatorname{Ass}(A)$ is nonempty. By contrast, Nakano [19] gives an example of a Prüfer domain such that no finitely generated ideal has a Zariski-Samuel associated prime.

The set $\operatorname{Ass}(A)$ behaves well with respect to localization:

Lemma 1.1. (Lemma 2.4(ii) of [4]) Let $A$ be an ideal of a ring $R$ and $P$ be a prime ideal of $R$ containing $A$. Then $P \in \operatorname{Ass}(A)$ if and only if $P_{M} \in \operatorname{Ass}\left(A_{M}\right)$ for some (or equivalently every) maximal ideal $M$ of $R$ containing $P$.

It is not hard to see that if $A$ is a proper ideal of a ring $R$, then every member of $\operatorname{Ass}(A)$ is contained in a maximal member of $\operatorname{Ass}(A)$. We define $\mathcal{X}_{A}$ to be the maximal members of $\operatorname{Ass}(A)$, that is, $\mathcal{X}_{A}$ consists of the maximal Krull associated primes of $A$. There exist examples of rings with ideals $A$ such that $\mathcal{X}_{A}$ is infinite. (See Example 2.6 of [4] for example, or use Lemma 1.5 below.) These examples are necessarily non-Noetherian.

In Lemma 2.3 of [4], we observe that $S(A)=\bigcup_{P \in \mathcal{X}_{A}} P$. In particular, $\bigcup_{P \in \mathcal{X}_{A}} P=$ $\bigcup_{Q \in \operatorname{Max}(A)} Q$. Despite this close connection between $\mathcal{X}_{A}$ and $\operatorname{Max}(A)$, one cannot conclude in general that $\mathcal{X}_{A} \subseteq \operatorname{Max}(A)$ or $\operatorname{Max}(A) \subseteq \mathcal{X}_{A}$. If $\operatorname{Max}(A)$ has only one member, then it is easy to see $\operatorname{Max}(A)=\mathcal{X}_{A}$. However, in Example 3.8 of [4] we construct a ring $R$ with Noetherian prime spectrum and an ideal $A$ of $R$ such that $\operatorname{Max}(A)$ has only two elements, but neither maximal prime divisor of $A$ is in $\mathcal{X}_{A}$. We also construct in Example 2.9 of [4] a 2-dimensional Prüfer domain $R$ for which $\operatorname{Spec}(R)$ is Noetherian and for which there exist an ideal $A$ and a finitely generated maximal ideal $M$ such that $M$ is a maximal prime divisor of $A$ and yet $M \notin \operatorname{Ass}(A)$.

When $R$ is arithmetical, the set $\operatorname{Ass}(A)$ has some striking properties. In the next sections we will need the following lemmas.

Lemma 1.2. (Proposition 2.7 of [4]) Let $R$ be an arithmetical ring and let $A$ be a proper ideal of $R$. If $P \in \operatorname{Ass}(A)$, then $Q \in \operatorname{Ass}(A)$ for every prime ideal $Q$ of $R$ such that $A \subseteq Q \subseteq P$. 
Corollary 1.3. Let $A$ be a proper ideal of an arithmetical ring $R$. Then $\mathcal{X}_{A}=$ $\operatorname{Max}(A)$ if and only if every prime ideal of $R$ that contains $A$ and is not prime to $A$ is a Krull associated prime of $A$.

Proof. Assume that $\mathcal{X}_{A}=\operatorname{Max}(A)$. If $P$ contains $A$ and is not prime to $A$, then $P$ is contained in some $Q \in \mathcal{X}_{A}$. Hence by Lemma 1.2, $Q \in \operatorname{Ass}(A)$. The converse is clear.

Let $A$ be a nonzero proper ideal of a Prüfer domain $R$ and let $Q$ be a prime ideal of $R$ with $A \subseteq Q$. Since $R_{Q}$ is a valuation domain, $\operatorname{End}\left(A R_{Q}\right)=R_{P}$ for some $P \in \operatorname{Spec} R$ with $P \subseteq Q$. Define

$$
\mathcal{E}_{A}=\left\{P \in \operatorname{Spec} R: R_{P}=\operatorname{End}\left(A R_{Q}\right) \text { for some prime ideal } Q \text { containing } A\right\} .
$$

Lemma 1.4. (Proposition 2.8 of [4]) Let $A$ be a nonzero proper ideal of a Prüfer domain $R$. Then $\operatorname{Ass}(A)=\mathcal{E}_{A}$. In particular, if $P \in \mathcal{X}_{A}$, then there exists a maximal ideal $M$ of $R$ such that $\operatorname{End}\left(A_{M}\right)=R_{P}$.

Lemma 1.5. Let $A$ be an ideal of an arithmetical ring $R$. If $A_{M}$ is a nonzero finitely generated ideal of $R_{M}$ for each maximal ideal $M$ of $R$, then $\operatorname{Ass}(A)$ is precisely the set of prime ideals of $R$ containing $A$.

Proof. First assume that $R$ is a valuation ring and $A$ is a nonzero finitely generated proper ideal of $R$. Then $A=y R$ is a principal ideal of $R$. Let $P$ be the maximal ideal of $R$. If $A=P$, then clearly $P \in \operatorname{Ass}(A)$, so suppose there exists $x \in P \backslash A$. Since $R$ is a valuation ring, $A \subset R x$ and $A=(A: x) x R$. If $(A: x)=A$, then $A=x A$ and $y=x y a$ for some $a \in A$. But this means $y(1-x a)=0$. Since $1-x a$ is a unit of $R$, this implies $y=0$, a contradiction to our assumption that $A \neq 0$. Therefore each $x \in P$ is non-prime to $A$, so $A$ is a primal ideal with $S(A)=P$ and $P \in \operatorname{Ass}(A)$. In the general case where $R$ is an arithmetical ring, we conclude from Lemma 2.1 and the preceding argument that every maximal ideal $P$ of $R$ that contains $A$ is in $\operatorname{Ass}(A)$. Hence by Lemma 1.2, every prime ideal of $R$ containing $A$ is in $\operatorname{Ass}(A)$.

\section{The CASE $\mathcal{X}_{A} \subseteq \operatorname{Max}(A)$}

In this section we characterize when $\mathcal{X}_{A} \subseteq \operatorname{Max}(A)$ for every regular ideal $A$ of an arithmetical ring. To do this, we first recall the notion of separated prime ideals, but we reformulate this definition to include rings with zero-divisors in such a way that our definition agrees with that of the separation property for domains (see pp. 91-92 of [3]). We define a ring $R$ to have the separation property if for each 
pair of distinct comparable regular prime ideals $P \subset Q$ of $R$, there exists a finitely generated ideal $A$ such that $P \subset A \subseteq Q$. The ring $R$ has the strong separation property if for each pair of comparable regular prime ideals $P \subset Q$ of $R$ and regular element $r \in P$, there exists $s \in Q$ such that $P \subset(r, s) R \subseteq Q$.

Clearly every one-dimensional domain has the strong separation property. By the Altitude Theorem of Krull [18, page 26] or [13, page 110], a Noetherian domain of dimension greater than two has the separation property but not the strong separation property. By contrast, Prüfer domains need not possess either separation property. The Prüfer domains that have the separation property have been well-studied (see, for example, pp. 91-92 of [3] and Lemma 2.7 of [20]). Note that a Bézout domain $R$ has the separation property if and only if $R$ has the strong separation property. More generally, a Prüfer domain with the QR-property ( so in particular a Prüfer domain with torsion Picard group ) has the separation property if and only if it has the strong separation property.

In the following lemma, we collect some technical characterizations of the separation property for Prüfer domains that will be needed in the next section.

Lemma 2.1. The following statements are equivalent for a Prüfer domain $R$.

(i) $R$ has the separation property.

(ii) For each nonzero prime ideal $P$ of $R, P$ is a maximal prime ideal of $\operatorname{End}(P)$.

(iii) For each nonzero prime ideal $P$ of $R, \operatorname{End}\left(P_{M}\right)=\operatorname{End}(P)_{M}$ for every maximal ideal $M$ of $R$.

(iv) For each nonzero nonmaximal prime ideal $P$ of $R$, if $\left\{M_{i}\right\}$ is the collection of maximal ideals of $R$ not containing $P$, then $R_{P} \subseteq\left(\bigcap_{i} R_{M_{i}}\right) R_{M}$ for each maximal ideal $M$ of $R$ containing $P$.

(v) For each nonzero ideal $A$ of $R$, if $M$ is a maximal ideal with $A \subseteq M$ and $P$ is a prime ideal such that $\operatorname{End}(A)_{M}=R_{P}$, then no element of $P$ is prime to $A$.

Proof. (i) $\Leftrightarrow$ (ii) Lemma 4.2 .38 of [3].

(i) $\Leftrightarrow$ (iii) Lemma 2.7 of $[20]$.

(iii) $\Rightarrow$ (iv) By Theorem 3.2.6 of [3] $\operatorname{End}(P)=R_{P} \cap\left(\bigcap_{i} R_{M_{i}}\right)$ and $\operatorname{End}\left(P_{M}\right)=$ $R_{P}$. Thus by (iii) $R_{P}=\operatorname{End}\left(P_{M}\right)=\operatorname{End}(P)_{M}=R_{P} \cap\left(\bigcap_{i} M_{i}\right) R_{M}$, and (iv) follows.

(iv) $\Rightarrow$ (v) Let $A$ be a nonzero ideal of $R$ and $M$ a maximal ideal with $A \subseteq M$. Let $P$ be a prime ideal such that $\operatorname{End}(A)_{M}=R_{P}$. It follows from Lemma 1.4 that

$$
\operatorname{End}(A)=\left(\bigcap_{Q \in \mathcal{X}_{A}} R_{Q}\right) \cap\left(\bigcap_{N} R_{N}\right)
$$


where $N$ ranges over the maximal ideals of $R$ that do not contain $A$. Let $Q^{\prime}$ be the unique member of $\mathcal{X}_{A}$ that is contained in $M$. By Lemma 1.4, $\operatorname{End}\left(A_{M}\right)=R_{Q^{\prime}}$. If $Q^{\prime}$ is a maximal ideal of $R$, then $Q^{\prime}=M$ and $\operatorname{End}\left(A_{M}\right)=R_{M}$, so $R_{P} \subseteq R_{M}$ implies that $M=P=Q^{\prime} \in \operatorname{Ass}(A)$, and the claim is clear. It remains to consider the case where $Q^{\prime}$ is a non-maximal prime ideal of $R$. Now

$$
R_{P}=\operatorname{End}(A)_{M}=\left(\bigcap_{Q \in \mathcal{X}_{A}} R_{Q}\right) R_{M} \cap\left(\bigcap_{N} R_{N}\right) R_{M} .
$$

We claim that $\bigcap_{Q \in \mathcal{X}_{A}} R_{Q} \subseteq R_{P}$. If this is not the case then since $R_{M}$ is a valuation domain it must be that $R_{P} \subsetneq\left(\bigcap_{Q \in \mathcal{X}_{A}} R_{Q}\right) R_{M}$. Hence from the above representation of $\operatorname{End}(A)_{M}$ we deduce that since $R_{P}$ is a valuation domain, $R_{P}=$ $\left(\bigcap_{N} R_{N}\right) R_{M}$. Thus $\left(\bigcap_{N} R_{N}\right) R_{M} \subsetneq\left(\bigcap_{Q \in \mathcal{X}_{A}} R_{Q}\right) R_{M}$. By (iv), $R_{Q^{\prime}} \subseteq\left(\bigcap_{N} R_{N}\right) R_{M}$ since no $N$ contains $Q^{\prime}$. However $Q^{\prime} \in \mathcal{X}_{A}$, so this implies $R_{Q^{\prime}} \subsetneq R_{Q^{\prime}} R_{M}$, but since $M$ contains $Q^{\prime}, R_{Q^{\prime}} R_{M}=R_{Q^{\prime}}$. This contradiction implies that $\bigcap_{Q \in \mathcal{X}_{A}} R_{Q} \subseteq R_{P}$, so every element $r \in P$ is contained in some $Q \in \mathcal{X}_{A}$. Consequently, no element of $P$ is prime to $A$.

(v) $\Rightarrow$ (iii) Suppose $P$ is a non-maximal prime ideal of $R$, and let $M$ be a maximal ideal of $R$ containing $P$. Let $Q$ be a prime ideal of $R$ such that $\operatorname{End}(P)_{M}=R_{Q}$. (Since $\operatorname{End}(P)_{M}$ is a valuation domain such a prime $Q$ must exist). Then by (v), the elements of $Q$ are not prime to $P$. Consequently, $P=Q$, and (iii) follows.

Using the strong separation property, we can characterize when $\mathcal{X}_{A} \subseteq \operatorname{Max}(A)$ for every regular ideal $A$ of an arithmetical ring. In fact, the strong separation property is always sufficient, regardless of whether $R$ is arithmetical, to guarantee that $\mathcal{X}_{A} \subseteq \operatorname{Max}(A)$ :

Lemma 2.2. If $A$ is a regular ideal of a ring $R$ having the strong separation property, then every $P \in \mathcal{X}_{A}$ is a maximal prime divisor of $A$, i.e., $\mathcal{X}_{A} \subseteq \operatorname{Max}(A)$.

Proof. Let $P \in \mathcal{X}_{A}$. There exists $Q \in \operatorname{Max}(A)$ such that $P \subseteq Q$. We show $P=Q$. If $\mathcal{X}_{A}$ consists only of $P$, then $A$ is $P$-primal and $P=Q$. Suppose $\mathcal{X}_{A} \backslash\{P\}$ is nonempty, and let $\left\{P_{i}\right\}=\mathcal{X}_{A} \backslash\{P\}$. Since $Q \in \operatorname{Max}(A), Q \subseteq P \cup\left(\bigcup_{i} P_{i}\right)$. Assume $P$ is properly contained in $Q$. Since $R$ has the strong separation property and $A$ is a regular ideal, there exists $x \in Q \backslash P$ such that $P \subset A+(x) \subseteq Q$. It follows that $x$ is in one of the $P_{i}$, so $P \subset A+(x) \subseteq P_{i}$. This contradicts the assumption that $P \in \mathcal{X}_{A}$ is maximal among the Krull associated primes of $A$. Thus $P=Q$.

Theorem 2.3. Let $R$ be an arithmetical ring. The following statements are equivalent.

(i) $R$ has the strong separation property. 
(ii) For each proper regular ideal $B$ of $R, \mathcal{X}_{B} \subseteq \operatorname{Max}(B)$.

Proof. By Lemma 2.2, (i) implies (ii). To prove that (ii) implies (i), suppose $P \subset Q$ are regular prime ideals of $R$ and that $a$ is a regular element with $a \in P$. Define $B=a P$ and note that the maximal ideals of $R$ that contain $B$ are precisely those that contain $a R$. We first show that $\mathcal{X}_{B}$ is the set union of $\{P\}$ and the set of maximal ideals of $R$ that contain $B$ but not $P$.

Since $a$ is a regular element, we have $P=B: a$, so $P \in \operatorname{Ass}(B)$. We claim that $P \in \mathcal{X}_{B}$. Suppose $L \in \mathcal{X}_{B}$ and $P \subseteq L$. Let $M$ be a maximal ideal containing $L$. By Lemma 1.7 of [4], $\left(B R_{M}\right)_{\left(P R_{M}\right)}$ is a $P_{M}$-primal ideal of $R_{M}$ since $B R_{M}$ is a finitely generated ideal of $R_{M}$. We claim $B R_{M}=\left(B R_{M}\right)_{\left(P R_{M}\right)}$. To verify that this is the case, we need only show that $B R_{M}: y=B R_{M}$ for all $y \in R_{M} \backslash P_{M}$. To this end, observe first that $P R_{M}=y P R_{M}$ for all $y \in R_{M} \backslash P_{M}$. This is because $R_{M}$ is a chained ring, so if $y \in R_{M} \backslash P_{M}$, then $P R_{M} \subseteq y R_{M}$, and if $p \in P R_{M}$, then $p=y u$ for some $u \in R_{M}$. Since $y \notin P R_{M}$, it follows that $u \in P R_{M}$ and $p \in y P_{M}$. Thus $P R_{M}=y P R_{M}$. Hence, with $y \in R_{M} \backslash P R_{M}$, we have that $B R_{M}: y=y B R_{M}:$ $y=B R_{M}$, since $y \notin P R_{M}$ implies that $P R_{M} \subseteq y R_{M}$ and hence that $y$ is a regular element of $R_{M}$. Therefore $B R_{M}=\left(B R_{M}\right)_{P R_{M}}$, and we conclude that $B R_{M}$ is a $P R_{M}$-primal ideal. However, by Lemma $1.1 L R_{M} \in \operatorname{Ass}\left(B R_{M}\right)$, so this forces $L R_{M} \subseteq P R_{M}$. Hence $L \subseteq P$, and we may conclude that $P=L \in \mathcal{X}_{B}$.

Now suppose $N$ is a maximal ideal of $R$ that contains $B$ but not $P$. Then $B_{N}=a R_{N} \neq 0$, so $N$ is in $\mathcal{X}_{B}$ by Lemma 1.5 and Lemma 1.1. This proves that the set $\mathcal{X}_{B}$ contains $P$ and the maximal ideals of $R$ that contain $B$ but not $P$. The reverse inclusion follows from the fact that any prime ideal of $R$ that is a member of $\mathcal{X}_{B}$ must contain $B$.

Let $\left\{N_{i}\right\}$ be the set of members of $\mathcal{X}_{B}$ distinct from $P$. As noted, each $N_{i}$ is a maximal ideal of $R$, and $\left\{N_{i}\right\}$ is precisely the set of maximal ideals of $R$ that contain $a R$ but not $P$. Since $P \in \mathcal{X}_{B}$ and, by (ii), $\mathcal{X}_{B} \subseteq \operatorname{Max}(B)$, we have $Q \nsubseteq S(B)=P \cup\left(\bigcup_{i} N_{i}\right)$, for otherwise $P$, as a proper subset of $Q$, would not be in $\operatorname{Max}(A)$. In particular, there exists $x \in Q$ such that $x$ is not contained in $P$ nor in any of the $N_{i}$. We have $(a, x) R \subseteq Q$, and to complete the proof we show that $P \subset(a, x) R$. It is enough by Theorem II.3.1 of [1, page 88] to show that $P_{M} \subset(A, x) R_{M}$ for all maximal ideals $M$ of $R$ that contain $(A, x)$. Observe that if $M$ is such a maximal ideal, then since by design $x$ is not an element of any maximal ideal of $R$ that contains $R a$ but not $P$, it must be that $M$ contains $P$. Now since $R_{M}$ is valuation ring, the ideals $P_{M}$ and $(a, x) R_{M}$ are comparable, so if $P_{M} \nsubseteq(a, x) R_{M}$, then it must be that $(a, x) R_{M} \subseteq P_{M}$. But then $x \in P_{M}$ and 
since $P \subseteq M$, it is the case that $P_{(M)}=P$, and we have $x \in P$, a contradiction to the choice of $x$. Thus we conclude that $P \subseteq(a, x) R \subseteq Q$, and $R$ has the strong separation property.

It follows from Theorem 2.3 that if $R$ is an arithmetical ring that does not have the strong separation property, then there exist a proper regular ideal $B$ of $R$ and $P \in \mathcal{X}_{B}$ such that $P \notin \operatorname{Max}(B)$. By definition of $\operatorname{Max}(B)$, this means there exists $Q \in \operatorname{Max}(B)$ such that $P$ is properly contained in $Q$ and by definition of $\mathcal{X}_{B}$, this means $Q \notin \operatorname{Ass}(B)$. Therefore the strong separation property is a necessary condition on an arithmetical ring in order that maximal prime divisors (of regular ideals) always be Krull primes.

Discussion 2.4. Some important examples of Prüfer domains do not have the separation property, and so by Theorem 2.3 , these Prüfer domains have ideals $B$ such that $\mathcal{X}_{B} \nsubseteq \mathbb{M a x}(B)$. Thus there exist maximal prime divisors of $B$ that are not in $\operatorname{Ass}(B)$. For example, neither the ring of entire functions nor the ring of integer-valued polynomials has the separation property. Indeed, both these rings are completely integrally closed integral domains of Krull dimension greater than one. If $P$ is a nonzero nonmaximal prime ideal of such a Prüfer domain $R$, then $\operatorname{End}(P)=R$ and $P$ is not maximal in $\operatorname{End}(P)$. Hence by Lemma 2.1 (ii), such domains do not have the separation property. On the other hand, every Prüfer domain having Noetherian maximal spectrum has the separation property (apply Theorem 4.2.39 of [3] and [22]).

\section{The CASE $\mathcal{X}_{A}=\operatorname{Max}(A)$}

Our main objective in this section is to investigate conditions on an arithmetical ring $R$ in order that $\mathcal{X}_{A}=\operatorname{Max}(A)$ for each regular ideal $A$ of $R$. We recall that an integral domain $R$ is a $Q R$-domain if every overring of $R$ is a localization of $R$ with respect to some multiplicatively closed subset of $R$. It is well-known that QR-domains are necessarily Prüfer, and that a Prüfer domain with torsion Picard group (e.g. a Bézout domain) is a QR-domain. More generally, a Prüfer domain $R$ is a QR-domain if and only if the radical of every finitely generated ideal of $R$ is the radical of a principal ideal of $R$ [21]. There exist QR-domains having nontorsion Picard group [9]. Thus the condition on a Prüfer domain $R$ that the radical of every finitely generated ideal is the radical of a principal ideal does not imply $R$ has torsion Picard group. However, it is clear that a QR-domain that has the separation property also has the strong separation property. 
Lemma 3.1. Let $A$ be an ideal of a Prüfer domain $R$. Suppose $Q$ is a prime ideal of $R$ that contains $A$, and $P$ is a prime ideal such that $\operatorname{End}(A)_{Q}=R_{P}$. If $P \in \operatorname{Ass}(A)$, then $\operatorname{End}(A)_{Q}=\operatorname{End}\left(A_{Q}\right)$.

Proof. Since $P \in \operatorname{Ass}(A), A_{(P)}$ is a primal ideal with adjoint prime $P$, and it follows that $A_{P}$ is a $P_{P}$-primal ideal. By Lemma 1.4, $\operatorname{End}\left(A_{P}\right)=R_{P}$. Thus $\operatorname{End}\left(A_{P}\right)=\operatorname{End}(A)_{Q}$, so $A \operatorname{End}\left(A_{P}\right)=A \operatorname{End}(A)_{Q}$ implies $A_{P}=A_{Q}$. Consequently, $\operatorname{End}\left(A_{Q}\right)=\operatorname{End}\left(A_{P}\right)=R_{P}=\operatorname{End}(A)_{Q}$.

Lemma 3.2. Let $R$ be a Prüfer domain with field of fractions $F$, let $X$ be an $R$ submodule of $F$, and let $M$ be a maximal ideal of $R$. Then $\operatorname{End}(X)_{M}=R_{P}$ for some $P \in \operatorname{Spec} R$ with $P \subseteq M$. Assume that $P$ is the union of prime ideals $P_{i}$, where each $P_{i}$ is the radical of a finitely generated ideal. Then $\operatorname{End}(X)_{Q}=\operatorname{End}\left(X_{Q}\right)$ for all prime ideals $Q$ such that $P \subseteq Q \subseteq M$.

Proof. Since $R_{M} \subseteq \operatorname{End}(X)_{M}$ and $R_{M}$ is a valuation domain, $\operatorname{End}(X)_{M}=R_{P}$ for some prime ideal $P \subseteq M$. If $\operatorname{End}(X)_{M}=F$, then clearly $\operatorname{End}(X)_{M}=\operatorname{End}\left(X_{M}\right)$, so we assume $\operatorname{End}(X)_{M} \neq F$ and thus $P \neq(0)$. Let $Q$ be a prime ideal of $R$ such that $P \subseteq Q \subseteq M$. Since $\operatorname{End}(X)_{M}=R_{P}$, we have $\operatorname{End}(X)_{Q}=R_{P}$. Now $R_{P}=\operatorname{End}(X)_{Q} \subseteq \operatorname{End}\left(X_{Q}\right) \subseteq \operatorname{End}\left(X_{P}\right)$, so to prove Lemma 3.2, it suffices to show that $\operatorname{End}\left(X_{P}\right) \subseteq R_{P}$.

Let $S=\operatorname{End}(X)$. Now $P S \subseteq P R_{P}$, so $P S \neq S$. Since $S$ is an overring of the Prüfer domain $R, S$ is a flat extension of $R$, so $P S$ is a prime ideal of $S$ and $S_{P S}=R_{P}$. Also, $P S$ is the union of the prime ideals $P_{i} S$, and each $P_{i} S$ is the radical of a finitely generated ideal of $S$.

Let $L$ be a prime ideal of $S$ such that $L \subseteq P S$ and such that $L=\sqrt{I}$, where $I$ is a finitely generated ideal of $S$. We prove there exists a nonzero $q \in F$ such that $q X_{L}$ is an ideal of $S_{L}$ that is primary for $L_{L}$. The invertible ideal $I^{2}$ of $S$ is an intersection of principal fractional ideals of $S$. Since $\operatorname{End}(X)=S$, each principal fractional ideal of $S$ is an intersection of $S$-submodules of $F$ of the form $q X, q \in F$. Since $I^{2} \subseteq L, I^{2}$ is an intersection of ideals of $S$ of the form $L \cap q X$, where $q \in F$. Since $I^{2} \subsetneq I \subseteq L$, there exists $q \in F$ such that $I^{2} \subseteq L \cap q X \subsetneq L$. Hence there exists a maximal ideal $N$ of $S$ with $I^{2} \subseteq N$ such that $I_{N}^{2} \subseteq L_{N} \cap q X_{N} \subsetneq L_{N}$. Since $S_{N}$ is a valuation domain, the $S_{N}$-modules $q X_{N}$ and $L_{N}$ are comparable and $I_{N}^{2} \subseteq L_{N} \cap q X_{N} \subsetneq L_{N}$ implies $I_{N}^{2} \subseteq q X_{N} \subsetneq L_{N}$. Now $\sqrt{I^{2}}=\sqrt{I}=L$ and $I^{2} \subseteq N$ implies $L \subseteq N$. Thus $I_{L}^{2} \subseteq q X_{L} \subseteq L_{L}$, and we conclude that $\sqrt{q X_{L}}=L_{L}$.

We observe next that $X_{P} \neq F$. Since $P \neq 0$, there exists a nonzero $L=P_{i} S \subseteq$ $P S$ such that $L=\sqrt{I}$, where $I$ is finitely generated. As we have established in the 
paragraph above, there exists a nonzero $q \in F$ such that $q X_{L}$ is an ideal of $S_{L}$. Thus $q X_{P} \subseteq q X_{L} \subseteq S_{L}$, so it is not possible that $X_{P}=F$.

Fix some member $L$ of the chain $\left\{P_{i} S\right\}$. Since $X_{P} \neq F, L \subseteq P S$ and $R_{P}$ is a valuation domain, there exists a nonzero element $s$ of $S$ such that $s X \subseteq L_{L}$. Since $\operatorname{End}\left(X_{P}\right)=\operatorname{End}\left(s X_{P}\right)$ and we wish to show that $\operatorname{End}\left(X_{P}\right) \subseteq R_{P}$ we may assume without loss of generality that $s=1$; that is, we assume for the rest of the proof that $X \subseteq L_{L}$. Define $A=X \cap S$. Then $A$ is an ideal of $S$. Moreover $A$ is contained in $L$ since $A_{L} \subseteq X_{L} \subseteq L_{L}$.

With the aim of applying Lemma 3.1, we observe that $P S \in \operatorname{Ass}(A)$. For each $i$ define $L_{i}=P_{i} S$. It suffices to show each $L_{i}$ with $L \subseteq L_{i} \subseteq P S$ is in $\operatorname{Ass}(A)$, since this implies that $P S=\bigcup_{L_{i} \supseteq L} L_{i}$ is a union of members of $\operatorname{Ass}(A)$. Let $i$ be such that $L \subseteq L_{i}$. Since $L_{i}$ is the radical of a finitely generated ideal of $S$, there exists (as we have established above) a nonzero $q \in F$ such that $q X_{L_{i}}$ is an ideal of $S_{L_{i}}$ that is primary for $\left(L_{i}\right)_{L_{i}}$. Now $A_{L_{i}}=X_{L_{i}} \cap S_{L_{i}}$. Since $S_{L_{i}}$ is a valuation domain, $A_{L_{i}}=X_{L_{i}}$ or $S_{L_{i}} \subseteq X_{L_{i}}$. By assumption, $X \subseteq L_{L}$. Since $L \subseteq L_{i}, X_{L_{i}} \subseteq L_{L}$, and it is impossible that $S_{L_{i}} \subseteq X_{L_{i}}$. Thus $A_{L_{i}}=X_{L_{i}}$. Consequently, $q X_{L_{i}}=q A_{L_{i}}$ and $q A_{L_{i}}$ is an ideal of $S_{L_{i}}$ that is primary for $\left(L_{i}\right)_{L_{i}}$. Since $S_{L_{i}}$ is a valuation domain, it follows that $q A_{L_{i}}=A_{L_{i}}: s$ for some $s \in S$. Thus $\left(L_{i}\right)_{L_{i}} \in \operatorname{Ass}\left(A_{L_{i}}\right)$, so by Lemma 1.1, $L_{i} \in \operatorname{Ass}(A)$. This proves $P S \in \operatorname{Ass}(A)$.

Since $A=X \cap S$ is an ideal of $S, S \subseteq \operatorname{End}(A)$. For each maximal ideal $N$ of $S$, either $A_{N}=X_{N}$ or $A_{N}=S_{N}$ It follows that $\operatorname{End}(A) \subseteq \operatorname{End}(X)=S$, so $\operatorname{End}(A)=S$. Thus $\operatorname{End}(A)_{P}=S_{P}=R_{P}$, and by Lemma 3.1, $\operatorname{End}\left(A_{P}\right)=R_{P}$. (We have used here that $S_{S P}=R_{P}$.) Now $A_{P}=X_{P} \cap S_{P}=X_{P} \cap R_{P}$. Since $R_{P}$ is a valuation domain, $A_{P}=X_{P}$ or $R_{P} \subseteq X_{P}$. The latter case is impossible since $X_{P} \subseteq X_{L} \subseteq L_{L}$. Thus $A_{P}=X_{P}$. We conclude that $\operatorname{End}\left(X_{P}\right)=\operatorname{End}\left(A_{P}\right)=$ $R_{P}$

A prime ideal $P$ is branched if there exists a $P$-primary ideal different from $P$. If $P$ is a nonzero prime ideal of a Prüfer domain $R$, then $P$ is branched if and only if $P$ is not the union of the prime ideals properly contained in $P$, and in this case if $P$ fails to be branched, then the valuation domain $R_{P}$ is infinite dimensional and there is no maximal element among the prime ideals properly contained in $P$.

Lemma 3.3. Let $R$ be a Prüfer domain having the separation property. If there exists a finitely generated ideal $J$ of $R$ having infinitely many minimal primes, then there exists a submodule $X$ of the field of fractions $F$ of $R$ and a maximal ideal $M$ containing $J$ such that $\operatorname{End}(X)_{M} \neq \operatorname{End}\left(X_{M}\right)$. 
Proof. Let $P_{1}, P_{2}, P_{3}, \ldots$ be countably many distinct minimal primes of $J$. Define $S=\bigcap_{i} R_{P_{i}}$. Then $S$ is a Prüfer overring of $R$ and the minimal primes of $J S$ are of the form $Q S$, where $Q \in \operatorname{Spec} R$ is a minimal prime of $J$. In particular, $P_{i} S$ is a minimal prime of $J S$ for each $i \geq 1$. Since $R$ has the separation property, each $P_{i} S$ is a maximal ideal of $S$. This is because if $j$ is any positive integer, then $\operatorname{End}\left(P_{j}\right)=R_{P_{j}} \cap\left(\bigcap_{N} R_{N}\right)$ by Theorem 3.2.6 of [3], so $\operatorname{End}\left(P_{j}\right) \subseteq S$ since the $P_{i}$ 's are comaximal. By Lemma 2.1(ii) $P_{j}$ is a maximal ideal of $\operatorname{End}\left(P_{j}\right)$, and since $R$ is a Prüfer domain, either $P_{j}$ extends to a maximal ideal $S P_{j}$ of $S$ or $S P_{j}=S$. The latter case is impossible since $S \subseteq R_{P_{j}}$. Thus $S P_{j}$ is a maximal ideal of $S$.

Since $J$ is a nonzero finitely generated ideal, each minimal prime of $J$ is branched. Since $J S$ has infinitely many minimal primes, Theorem 1.6 of [7] implies there exists a minimal prime $Q S$ of $J S$ that is not the radical of a finitely generated ideal. If $Q=P_{j}$ for some $j$, then by [6, Theorem 2], we have $S=\bigcap_{i \neq j} R_{P_{i}}$. Therefore, by relabeling if necessary, we may assume that $Q \notin\left\{P_{i}\right\}_{i=1}^{\infty}$.

Define $A=J R_{Q} \cap R$. Then $A S=J R_{Q} \cap S$ is $Q S$-primary. In particular, $Q S$ is the unique minimal prime of $A S$ and $A \nsubseteq P_{i} S \cap R=P_{i}$ for each $i \geq 1$.

Let $Q_{1}$ be the prime ideal of $R$ just below $Q$ (such a prime $Q_{1}$ exists because $Q$ is branched). Since $R$ has the separation property, there is a finitely generated ideal $B$ of $R$ such that $Q_{1} \subset B \subseteq Q$. Local verification shows that $Q_{1} \subset B^{n} \subseteq Q$ for each positive integer $n$.

For each $i \geq 1$, let

$$
x_{i} \in A^{i} \backslash\left(P_{1} \cup \cdots \cup P_{i} \cup A^{i+1}\right) .
$$

(By Prime Avoidance (see for example [2, Lemma 3.3]) such an element $x_{i}$ exists.) Define

$$
J_{i}=B^{i+1}+x_{i} R
$$

Then $Q_{1} \subseteq J_{i}$ and $J_{i} \not \subseteq\left(P_{1} \cup \cdots \cup P_{i}\right)$. Define $X=\sum_{i>1} J_{i}^{-1} S$.

Let $N=Q S$ and for each $i>0$, define $N_{i}=P_{i} S$. As previously demonstrated, $N$ and the ideals $N_{i}, i>0$, are maximal ideals of $S$. We show first that $\operatorname{End}\left(X_{N}\right) \neq$ $\operatorname{End}(X)_{N}$.

For each $i \geq 1$,

$$
X_{N_{i}}=\sum_{k=1}^{i-1} J_{k}^{-1} S_{N_{i}},
$$

since by design, $J_{k}^{-1} S_{N_{k}}=S_{N_{k}}$ for all $k \geq i$. So as a finitely generated fractional ideal of $S_{N_{i}}, X_{N_{i}}$ is isomorphic to $S_{N_{i}}$. Thus $\operatorname{End}\left(X_{N_{i}}\right)=S_{N_{i}}$ for each $i$. We have 
that for each $i, S_{N_{i}}=R_{P_{i}}$; hence

$$
\operatorname{End}(X) \subseteq \bigcap_{i} \operatorname{End}\left(X_{N_{i}}\right)=S
$$

Therefore, $\operatorname{End}(X)=S$. In particular, $\operatorname{End}(X)_{N}=S_{N}$.

Thus to prove the claim that $\operatorname{End}(X)_{N} \neq \operatorname{End}\left(X_{N}\right)$, it suffices to show that $N \operatorname{End}\left(X_{N}\right)=\operatorname{End}\left(X_{N}\right)$. To this end we verify that $X_{N}=N X_{N}$. Since $N=S Q$, we have $S_{N}=R_{Q}$, so for each $i \geq 1, A^{i+1} S_{N} \subset A^{i} S_{N}$. In particular, $x_{i+1} S_{N} \subset$ $x_{i} S_{N}$ since $x_{i} \in A^{i} \backslash A^{i+1}$ and $A^{i+1} S_{N} \cap R=A^{i+1} R_{Q} \cap R=A^{i+1}$. It follows that $\left(J_{i+1}\right) S_{N} \subset\left(J_{i}\right) S_{N}$. Since $J_{i}$ is a finitely generated ideal of $S$, it follows that $\left(J_{i+1}\right) S_{N} \subseteq N\left(J_{i}\right) S_{N}$. Consequently, $J_{i}^{-1} \subseteq N\left(J_{i+1}^{-1}\right) S_{N}$ for each $i \geq 1$. Therefore $X \subseteq N X_{N}$, as claimed.

For $S$-submodules $Y$ and $W$ of $F$, let $[W: Y]=\{q \in F: q Y \subseteq W\}$. Since $X_{N}=N X_{N}$ and $\operatorname{End}\left(N_{N}\right)=S_{N}$, we have

$$
\left[S_{N}: X_{N}\right]=\left[N_{N}: N X_{N}\right]=\left[N_{N}: X_{N}\right] .
$$

Moreover, $\left[S_{N}: X_{N}\right]=\bigcap_{i} J_{i} S_{N}$ and

$$
Q_{1} S_{N} \subseteq \bigcap_{i} J_{i} S_{N} \subseteq \bigcap_{i} A^{i} S_{N}=Q_{1} S_{N}
$$

The latter equality follows from the fact that since $S_{N}$ is a valuation domain and $A S_{N}$ is a principal ideal of $S_{N}$, the intersection $\bigcap_{i} A^{i} S_{N}$ is a prime ideal. Since $Q_{1}$ is the largest prime ideal of $R$ properly contained in $Q, A \subseteq Q$ and $A \nsubseteq Q_{1}$, we have $\bigcap_{i} A^{i} S_{N}=Q_{1} S_{N}$.

It follows that $\left[N_{N}: X_{N}\right]=\left[S_{N}: X_{N}\right]=\left(S Q_{1}\right)_{N}$. Since $R_{N}$ is a valuation domain, the maximal ideal $N_{N}$ of $S_{N}$ is $m$-canonical (see [10]), that is,

$$
X_{N}=\left[N_{N}:\left[N_{N}: X_{N}\right]\right]=\left[N_{N}:\left(S Q_{1}\right)_{N}\right] .
$$

Since $S Q_{1}$ is a nonmaximal prime ideal of $S,\left[N_{N}:\left(S Q_{1}\right)_{N}\right]=S_{S Q_{1}}[3$, Theorem 4.1.21]. Thus $X_{N}=S_{S Q_{1}}$, and we have $\operatorname{End}\left(X_{N}\right)=S_{S Q_{1}}=R_{Q_{1}}$. In particular, $\operatorname{End}\left(X_{N}\right) \neq R_{Q}$. Now $X_{N}=X_{S Q}=X_{Q}$, and if $M$ is a maximal ideal of $R$ containing $Q$, then $S_{S Q}=S_{M}$, so $X_{M}=X_{Q}$. Thus

$$
R_{Q_{1}}=\operatorname{End}\left(X_{N}\right)=\operatorname{End}\left(X_{M}\right)
$$

while

$$
\operatorname{End}(X)_{M}=S_{M}=R_{Q} .
$$

Therefore $\operatorname{End}\left(X_{M}\right) \neq \operatorname{End}(X)_{M}$.

Theorem 3.4. Let $R$ be a Prüfer domain with field of fractions $F$. The following statements are equivalent. 
(i) For each finitely generated ideal $A$ of $R$, every weak-Bourbaki associated prime of $A$ is a Zariski-Samuel associated prime of $A$.

(ii) Every finitely generated ideal of $R$ has only finitely many minimal prime ideals.

(iii) Every principal ideal of $R$ has only finitely many minimal prime ideals.

(iv) Every branched prime ideal of $R$ is the radical of a finitely generated ideal.

(v) For each $R$-submodule $X$ of $F, \operatorname{End}\left(X_{M}\right)=\operatorname{End}(X)_{M}$, for every maximal ideal $M$ of $R$.

Proof. (i) $\Rightarrow$ (ii) Let $A$ be a finitely generated ideal of $R$. Every minimal prime $P$ of $A$ is a weak-Bourbaki prime of $A$ and therefore by (i) a Zariski-Samuel prime of $A$ and hence of the form $\sqrt{(A: x)}$. Since $R$ is Prüfer, $(A: x)$ is finitely generated, see, for example, [6, Lemma 2]. Thus each minimal prime of $R / A$ is the radical of a finitely generated ideal of $R / A$. By Theorem 1.6 in [7], $R / A$ has finitely many minimal primes. This proves (ii).

(ii) $\Rightarrow$ (iii) is obvious.

(iii) $\Rightarrow$ (iv) Let $P$ be a branched prime of $R$. Then there exists a prime ideal $Q$ of $R$ such $Q \subset P$ and there are no other prime ideals between $Q$ and $P$. Let $x \in P \backslash Q$. Since the prime ideals contained in $P$ are linearly ordered, $P$ is a minimal prime ideal of $x R$. By (iii), $x R$ has only finitely many minimal prime ideals. Since $R$ is Prüfer, if $B$ is the intersection of the other minimal primes of $x R$, then $B+P=R$. Hence there exists $y \in P$ such that $B+y R=R$. It follows that $P$ is the radical of $(x, y) R$. This proves (iv).

(iv) $\Rightarrow$ (v) Since every nonzero prime ideal of $R$ is the union of branched prime ideals, this follows from Lemma 3.2.

(v) $\Rightarrow$ (i) Let $A$ be a finitely generated ideal of $R$ and let $P$ be a weak-Bourbaki associated prime of $A$. Then $P$ is a minimal prime of $(A: x)$ for some $x \in R$. Since $R$ is Prüfer, $(A: x)$ is finitely generated. Lemma 3.3 implies that $(A: x)$ has only finitely many minimal primes. Thus by Lemma 5.10 of [4] each minimal prime of $(A: x)$ is a Zariski-Samuel associated prime of $(A: x)$, and therefore a Zariski-Samuel associated prime of $A$. This proves (i).

There is an interesting connection between the conditions of Theorem 3.4 and "trace properties" of Prüfer domains. T. Lucas shows in [16, Theorem 23] that a Prüfer domain $R$ satisfies (iv) of Theorem 3.4 if and only if $R$ has the radical trace property, namely, for every ideal $A$ of $R, A A^{-1}$ is either $R$ or a radical ideal of $R$.

Corollary 3.5. The following statements are equivalent for a Prüfer domain $R$. 
(i) Every branched prime ideal of $R$ of height greater than one is the radical of a finitely generated ideal of $R$.

(ii) For each nonzero ideal $A$ of $R, \operatorname{End}\left(A_{M}\right)=\operatorname{End}(A)_{M}$ for all maximal ideals $M$ of $R$.

(iii) For each ideal $A$ of $R, \operatorname{Ass}(A)$ is precisely the set of prime ideals $P$ such that $A \subseteq P$ and $P \operatorname{End}(A) \neq \operatorname{End}(A)$.

Proof. (i) $\Rightarrow$ (ii) Suppose (i) holds, and let $A$ be a nonzero ideal of $R$. Let $M$ be a maximal ideal of $R$. If $M$ has height one then $R_{M}$ is a one-dimensional valuation domain, so $\operatorname{End}\left(A_{M}\right)=R_{M}$. Consequently, $\operatorname{End}(A)_{M}=\operatorname{End}\left(A_{M}\right)$. If $M$ has height greater than 1 , then $M$ is the union of branched prime ideals of height greater than 1 , so by (i) and Lemma $3.2, \operatorname{End}\left(A_{M}\right)=\operatorname{End}(A)_{M}$. This proves (ii).

(ii) $\Rightarrow$ (i) Let $P$ be a branched prime ideal of $R$ such that there is a nonzero prime ideal $Q$ of $R$ with $Q \subset P$. From (ii) and Lemma 2.1, it follows that $R$ has the separation property. Thus $Q$ is a maximal ideal of $\operatorname{End}(Q)$ and $\operatorname{End}(Q) / Q$ is the quotient field of $R / Q$. From (ii) it follows that if $X$ is an $R$-submodule of $\operatorname{End}(Q)$ with $Q \subseteq X$, then

$$
\operatorname{End}\left((X / Q)_{M / Q}\right)=\operatorname{End}(X / Q)_{M / Q}
$$

for all maximal ideals $M$ of $R$ containing $Q$. By Theorem 3.4, the branched prime ideal $P / Q$ of $R / Q$ is the radical of a finitely generated ideal. Hence $P$ is the radical of an ideal $I+Q$, where $I$ is a finitely generated ideal of $R$. Since $R$ has the separation property, there is a finitely generated ideal $J$ such that $Q \subset J \subseteq P$. Thus $P$ is the radical of the finitely generated ideal $I+J$.

(ii) $\Rightarrow$ (iii) Let $A$ be an ideal of $R$, and suppose $P \in \operatorname{Ass}(A)$. Then there exists $Q \in \mathcal{X}_{A}$ such that $P \subseteq Q$. By Lemma 1.4, $R_{Q}=\operatorname{End}\left(A_{M}\right)$ for some maximal ideal $M$ of $R$. Thus by (ii), $R_{Q}=\operatorname{End}(A)_{M}$, and we have $Q \operatorname{End}(A) \neq \operatorname{End}(A)$. It follows that $P \operatorname{End}(A) \neq \operatorname{End}(A)$.

Now suppose $P$ is a prime ideal containing $A$ such that $P \operatorname{End}(A) \neq \operatorname{End}(A)$. Then $\operatorname{End}(A)_{M} \neq P \operatorname{End}(A)_{M}$ for some maximal ideal $M$ of $R$, and it follows that $\operatorname{End}(A)_{M} \subseteq R_{P}$. By (ii), $\operatorname{End}\left(A_{M}\right) \subseteq R_{P}$. If $\operatorname{End}\left(A_{M}\right)=R_{Q}$ for some prime ideal $Q$, then by Lemma 1.2 and 1.4, it follows that $Q \in \operatorname{Ass}(A)$. Since $A \subseteq P \subseteq Q$, Lemma 1.2 implies $P \in \operatorname{Ass}(A)$. This proves (iii).

(iii) $\Rightarrow$ (ii) Let $A$ be a proper ideal of $R$ and $M$ be a maximal ideal of $R$ containing $A$. If $\operatorname{End}(A)_{M}=R_{P}$ for some prime ideal $P$ of $R$, then $P \operatorname{End}(A) \neq \operatorname{End}(A)$, so by (iii), $P \in \operatorname{Ass}(A)$. By Lemma $3.1, \operatorname{End}(A)_{M}=\operatorname{End}\left(A_{M}\right)$. This proves (ii). 
Remark 3.6. Theorem 3.4 shows that conditions for good behavior with respect to localization for endomorphism rings of submodules of the fraction field involve all the primes of $R$. On the other hand, Corollary 3.5 shows that the corresponding property for ideals involves only conditions on the primes of height greater than one. This phenomenon is illustrated in the article Goeters-Olberding [8], where the statement of Corollary 3.5 that $\operatorname{End}(A)$ commutes with localizations is of central importance. Our proof of Lemma 3.3 is a refinement of the argument given in Theorem 3.7 of [8].

Theorem 3.7. Consider the following statements for an arithmetical ring $R$.

(i) $\mathcal{X}_{A}=\operatorname{Max}(A)$ for each proper regular ideal $A$ of $R$.

(ii) Every branched prime ideal of $R$ that properly contains a regular prime ideal is the radical of a finitely generated ideal.

(iii) Every branched prime ideal of $R$ that properly contains a regular prime ideal is the radical of a principal ideal.

Then (iii) $\Rightarrow$ (i) $\Rightarrow$ (ii). Moreover, if $R$ is a $Q R$-domain, then all three statements are equivalent.

Proof. (i) $\Rightarrow$ (ii) We consider first the case where $R$ is a domain. Then to prove (ii), it suffices by Corollary 3.5 to show that for every ideal $A$ of $R$ and maximal ideal $M$ containing $A, \operatorname{End}(A)_{M}=\operatorname{End}\left(A_{M}\right)$. Let $A$ be a proper nonzero ideal of $R$ and let $M$ be a maximal ideal such that $A \subseteq M$. Since $R_{M}$ is a valuation domain, there exists a prime ideal $P$ of $R$ such that $\operatorname{End}(A)_{M}=R_{P}$. We claim first that the elements of $P$ are not prime to $A$. Let $\left\{M_{\alpha}\right\}$ denote the set of maximal ideals of $R$ that contain $A$, and let $\left\{N_{\beta}\right\}$ denote the set of maximal ideals of $R$ that do not contain $A$. For each $\alpha$, there exists a prime ideal $P_{\alpha}$ such that $\operatorname{End}\left(A_{M_{\alpha}}\right)=R_{P_{\alpha}}$. Moreover, by Lemmas 1.2 and 1.4, we have $\mathcal{X}_{A} \subseteq\left\{P_{\alpha}\right\} \subseteq \operatorname{Ass}(A)$. Since $\operatorname{End}(A)=$ $\left(\bigcap_{\alpha} \operatorname{End}\left(A_{M_{\alpha}}\right)\right) \cap\left(\bigcap_{\beta} \operatorname{End}\left(A_{N_{\beta}}\right)\right)$, we have

$$
R_{P}=\operatorname{End}(A)_{M}=\left(\bigcap_{\alpha} R_{P_{\alpha}}\right) R_{M} \cap\left(\bigcap_{\beta} R_{N_{\beta}}\right) R_{M} .
$$

Since $R$ has the separation property, Lemma 2.3(iv) implies that $\bigcap_{\beta} R_{N_{\beta}} \nsubseteq R_{P}$. Thus because $R_{P}$ is a valuation domain, it must be that $\bigcap_{\alpha} R_{P_{\alpha}} \subseteq R_{P}$, and it follows that $P \subseteq \cup_{\alpha} P_{\alpha}$. Since $S(A)=\cup_{\alpha} P_{\alpha}$, the elements of $P$ are not prime to $A$, as claimed. Therefore there exists $Q \in \operatorname{Max}(A)$ such that $P \subseteq Q$. By (i), $Q \in \mathcal{X}_{A}$, so by Lemma 1.2, $P \in \operatorname{Ass}(A)$ since $A \subseteq P \subseteq Q$. Thus by Lemma 3.1, $\operatorname{End}(A)_{M}=\operatorname{End}\left(A_{M}\right)$. This proves that (i) implies (ii) in the case $R$ is a domain.

Now consider the general case where $R$ is not necessarily a domain. Observe that since statement (i) holds for $R$, statement (i) holds for $R / P$ for all regular 
prime ideals $P$ of $R$. This follows from the observation that if $A$ is an ideal of $R$ that contains $P$ then for all $x \in R$,

$$
\left(A / P:_{R / P} x+P\right)=((A: x)+P) / P .
$$

In particular, $\operatorname{Ass}(A / P)=\{Q / P: Q \in \operatorname{Ass}(A), P \subseteq Q\}$. Also, $\operatorname{Max}(A / P)=$ $\{Q / P: Q \in \operatorname{Max}(A), P \subseteq Q\}$. Thus statement (ii) holds for $R / P$ for all regular prime ideals $P$ of $R$. Let $Q$ be a branched prime ideal of $R$ containing at least one regular prime ideal. Then there exists a regular prime ideal $P$ of $R$ such that $P \subset Q$. By Theorem 2.3, there exists a finitely generated ideal $B$ of $R$ such that $P \subseteq B \subset Q$. Moreover, since (ii) holds for $R / P$, there exists a finitely generated ideal $C$ of $R$ such that $Q$ is the radical of $C+P$. It follows that $Q$ is the radical of the finitely generated ideal $C+B$.

(iii) $\Rightarrow$ (i) Let $A$ be a proper regular ideal of $R$. For each $P \in \mathcal{X}_{A}$ there exists $M \in \operatorname{Max}(A)$ such that $P \subseteq M$ and then $P=M$ if and only if $M \in \operatorname{Ass}(A)$. Thus it suffices to prove that $M \in \operatorname{Max}(A)$ implies $M \in \operatorname{Ass}(A)$. This is clear if $M / A$ has height one as prime ideal of $R / A$. Assume that $\operatorname{ht}(M / A) \geq 2$. Then $M$ is the union of a chain of branched prime ideals $P_{i}$ of $R$, where $A \subseteq P_{i}$ and $\operatorname{ht}\left(P_{i} / A\right) \geq 2$. Since $A$ is a regular ideal, so is each $P_{i}$. Thus by (iii), each $P_{i}$ is the radical of a principal ideal of $R$. Since $P_{i} \subseteq M$, the elements in $P_{i}$ are non-prime to $A$. Since

$P_{i}$ is the radical of a principal ideal and $S(A)=\bigcup_{Q \in \mathcal{X}_{A}} Q, P_{i}$ is contained in some member of $\mathcal{X}_{A}$. By Lemma 1.2, each $P_{i} \in \operatorname{Ass}(A)$. Therefore $\bigcup_{i} P_{i}=M \in \operatorname{Ass}(A)$.

Finally, we conclude that statements (i),(ii) and (iii) are equivalent for a QRdomain $R$ since in a QR-domain every prime ideal that is the radical of a finitely generated ideal is the radical of a principal ideal.

Corollary 3.8. If $R$ is a Prüfer domain having the property that every maximal prime divisor of an ideal $A$ of $R$ is a Krull associated prime of $A$, then every branched prime ideal of $R$ of height greater than one is the radical of a finitely generated ideal.

Proof. The corollary is a consequence of Theorem 3.7 and Corollary 1.3.

Example 3.9 illustrates the fact that it is possible for a 2-dimensional Prüfer domain to satisfy the hypotheses of Theorem 2.3, but not Theorem 3.7.

Example 3.9. There exists a Prüfer domain $R$ that has the strong separation property and yet contains an ideal $A$ such that $\mathcal{X}_{A}$ is a proper subset of $\operatorname{Max}(A)$. Moreover, $R$ does not satisfy any of the conditions (i), (ii) or (iii) of Theorem 3.7. Let $D$ be an almost Dedekind domain (that is, $D_{M}$ is a DVR for all maximal ideals 
$M$ of $D$ ) that is not Dedekind. (See [3, page 281] for an example of such a domain.) Let $F$ denote the field of fractions of $D$, let $x$ be an indeterminate over $F$, and let $P=x F[x]_{(x)}$. Define $R=D+P$. Then $R$ is a Prüfer domain and $P$ is the unique nonzero nonmaximal prime ideal of $R$. Moreover, if $M$ is a maximal ideal of $R$, then $M$ contains $P$ and if $r \in M \backslash P$, then $P \subseteq R r \subseteq M$, so $R$ has the strong separation property. In particular, by Theorem 2.3, every ideal $A$ of $R$ has the property that $\mathcal{X}_{A} \subseteq \operatorname{Max}(A)$. Since $D$ is almost Dedekind, but not Dedekind, there exists a maximal ideal $Q$ of $D$ that is not the radical of a finitely generated ideal. Consequently, the (height 2) maximal ideal $Q+P$ of $R$ is not the radical of a finitely generated ideal of $R$. By Theorem 3.7, there exists an ideal $A$ of $R$ such that $\mathcal{X}_{A}$ is a proper subset of $\operatorname{Max}(A)$.

In Example 2.9 of [4], we construct a 2-dimensional Prüfer domain with Noetherian spectrum having an ideal $A$ for which there exists a maximal ideal $M$ such that $M$ is in $\operatorname{Max}(A)$ but $M$ is not a Krull associated prime of $A$. Since this domain $R$ has Noetherian spectrum, $R$ satisfies statement (ii) of Theorem 3.7. Hence condition (ii) of Theorem 3.7 does not imply condition (i), even when $R$ is a 2-dimensional Prüfer domain. This leaves the question of whether in general (i) implies (iii) in Theorem 3.7:

Question 3.10. Does there exist an arithmetical ring $R$ such that $R$ satisfies statement (i) but not statement (iii) of Theorem 3.7?

\section{REFERENCES}

[1] N. Bourbaki, Commutative Algebra, Chapters 1 - 7, Springer-Verlag, 1989.

[2] D. Eisenbud, Commutative algebra with a view toward algebraic geometry, Springer-Verlag, 1994.

[3] M. Fontana, J. Huckaba and I. Papick, Prüfer domains, Marcel Dekker, 1998.

[4] L. Fuchs, W. Heinzer and B. Olberding, Commutative ideal theory without finiteness conditions: primal ideals, to appear.

[5] R. Gilmer, A counterexample to two conjectures in ideal theory, Amer. Math. Monthly, 74 (1967), 195-197.

[6] R. Gilmer and W. Heinzer, Overrings of Prüfer domains II, J. Algebra 7 (1967), 281-302.

[7] R. Gilmer and W. Heinzer, Primary ideals with finitely generated radical in a commutative ring, Manuscripta Math. 78 (1993), 201-221.

[8] H. P. Goeters and B. Olberding, Extension of ideal-theoretic properties of a domain to its quotient field, J. Algebra 237 (2001), 14-31.

[9] W. Heinzer, Quotient overrings of integral domains, Mathematika 17 (1970), 139-148.

[10] W. Heinzer, J. Huckaba and I. Papick, m-Canonical ideals in integral domains, Comm. Algebra 26 (1998), 3021-3043.

[11] W. Heinzer and J. Ohm, Locally Noetherian commutative rings, Trans. Amer. Math. Soc., 158 (1971), 273-284.

[12] J. Iroz and D. Rush, Associated prime ideals in non-Noetherian rings, Can. J. Math. 36 (1984), 344-360.

[13] I. Kaplansky, Commutative Rings (University of Chicago Press, 1974). 
[14] W. Krull, Idealtheorie in Ringen ohne Endlichkeitsbedingung, Math. Ann. 101 (1929), 729744.

[15] W. Krull, Idealtheorie, Ergebnisse d. Math. (Berlin, 1935).

[16] T. Lucas, The radical trace property and primary ideals, J. Algebra 184 (1996), 1093-1112.

[17] M. Nagata, Some remarks on prime divisors, Memoirs College of Science, Kyoto University, Series A, 33 (1960) 297-299.

[18] M. Nagata, Local Rings (Interscience, 1962).

[19] N. Nakano, Idealtheorie in einem speziellen unendlichen algebraischen Zahlkörper, J. Sci. Hiroshima Univ. Ser. A 16 (1953), 425-439.

[20] B. Olberding, Globalizing local properties of Prüfer domains, J. Algebra 205 (1998), 480-504.

[21] R. Pendleton, A characterization of Q-domains, Bull. Amer. Math. Soc. 72 (1966), 499-500.

[22] D. Rush and L. Wallace, Noetherian maximal spectrum and coprimely packed localizations of polynomial rings, Houston J. Math. 28 (2002) 437-448.

Department of Mathematics, Tulane University, New Orleans, Louisiana 70118

E-mail address: fuchs@tulane.edu

Department of Mathematics, Purdue University, West Lafayette, Indiana 47907

E-mail address: heinzer@math.purdue.edu

Department of Mathematical Sciences, New Mexico State University, Las Cruces, NEw MEXiCO 88003-8001

E-mail address: olberdin@emmy.nmsu.edu 